\title{
A Note on Uncertainty in Real-Time Analytics
}

\author{
Mladen A. Vouk \\ Department of Computer Science \\ North Carolina State University \\ Raleigh, NC, USA \\ vouk@csc.ncsa.edu
}

\begin{abstract}
Today real-time analytics of large data sets is invariably computer-assisted and often includes a "human-in-the-loop". Humans differ from each other and all have a very limited innate capacity to process new information in real-time. This introduces statistical and systematic uncertainties into observations, analyses and decisions humans make when they are "in the loop". Humans also have unconscious and conscious biases, and these can introduce (major) systematic errors into human assisted or human driven analytics. This note briefly discusses the issues and the (considerable) implications they can have on real-time analytics that involves humans, including software interfaces, learning, and reaction of humans in emergencies.
\end{abstract}

Keywords: human mind bandwidth, uncertainty, human bias, real-time analytics, computer-assisted analytics, human-in-the-loop.

\section{Introduction}

True artificial intelligence is still the "stuff" of science fiction stories and possibly future. To avoid and mitigate mistakes in computer-driven modeling and decision making we often include a human into "the loop" — for example, FAA flight controllers, real-time network and computer security threat identification and mitigation analysts, inclement weather decision making personnel, or scientists involved in very expensive high-end simulations, e.g., [1. Well designed complex computer analytics and decision making systems, and workflows, include points where humans are inserted into the process to monitor, augment, direct, take over, or stop processes. This makes a lot of sense, and this can also be an issue. People have biases [2] and people make mistakes too, lots of them. An interesting, and often used alternative to "human-in-the-loop" is the "computer-in-the-loop" where analytics is human-driven and the computer only augments human decision making by offering suggestions, second opinions, etc. A recent well publicized example of this approach is the IBM's Watson project and its application in medicine [3].

This note provides an overview of some of the uncertainties that humans can inject into data analysis and interpretations processes. Sect. 2 of this note discusses information processing limitation of human mind in the context of new inputs, and in Sect. 3 it very briefly covers some possible systematic biases. Sect. 4 concludes the note.

A. Dienstfrey and R.F. Boisvert (Eds.): WoCoUQ 2011, IFIP AICT 377, pp. 312-318, 2012.

(C) IFIP International Federation for Information Processing 2012 


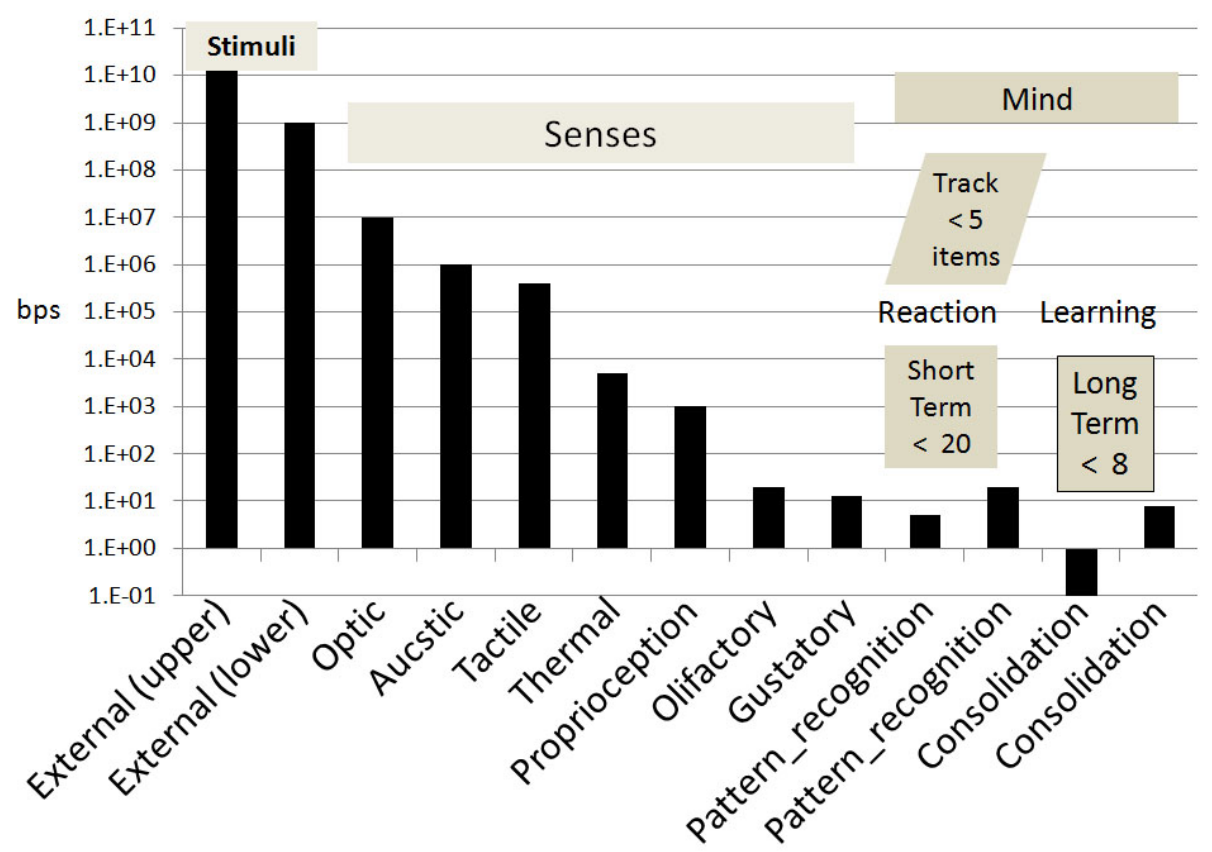

Fig. 1. Input to humans - data reduction is in the giga-fold range (after 4])

\section{Uncertainties}

From the perspective of external inputs, a human operates in five principal outward facing sensory domains - visual, auditory, tactile, olfactory, and taste, as well as in a number of inward-facing sensory domains 4 . There are a number of models of how sensory inputs end-up being processed, become actionable and possibly become memories, and there is a huge amount of literature regarding the human mind, its capabilities, and its limits. An interesting top-level general book on the topic of memory is by Thompson and Madigan [5].

\subsection{Information Flux}

Humans are bombarded with a huge amount of stimuli - as much as a terabit per second [4. Fig. 1 illustrates the information flow rates humans might receive and the principal stages of the related information processing. The scale on the vertical axis is logarithmic, and in units of bits per second (bps). Horizontal axis covers some of the basic senses, or sensors, humans have. Estimated upper and lower bounds are shown for the overall external flux of stimuli, and for short and long term memory information processing. Also shown are approximate maximum rates into different senses.

Humans do not process outside stimuli directly but through a multitude of sensor channels and filters which prepare that information for input into human 
mind. For example, optic channel may carry as much as 10 megabits per second, acoustic channel as much as a megabit per second, tactile channel is somewhat below that, thermal is in the range of several kilobits per second, as is the proprioception channel (position of limbs and body), while olfactory and gustatory channels are in the 10 to 20 bits per second range.

\subsection{Information Processing Capacity}

While a human brain has considerable capacity, and has very high internal processing speeds, conscious processing (pattern matching) of information a person needs to act upon (either physically or mentally) is considerable slower. Information that needs to be actively processed lands in short-term memory where it is then processed at the rate of 5 to 20 bits per second. Long-term storing of information (learning), happens at an even slower rate - from zero to perhaps 8 bits per second.

It is obvious that a considerable reduction needs to take place between inputs and the amount of new data a person "works with". This means that a lot of that data is either discarded, or is dynamically codified into patterns that a human acts upon (based on stored patterns) or stores permanently. The question is whether there is a loss of useful information in the process. If the processing is done automatically before presenting data to a human, there could be unintended loss of information that may bias the outcomes.

In addition, humans often have built-in biases brought on by culture, training, education, social environments, intent, and so on, and that can exacerbate the problem.

\section{$2.3 \quad$ Models}

As already mentioned, there are a number of models of how sensory inputs endup being processed, become actionable and possibly become memories. There is a huge amount of literature regarding the human mind, its capabilities, and its limits. For example, one model states that environmental input goes to sensors (vision, auditory, ...), then to short-term temporary working memory, and depending on what happens there, this "chunk" of information may elicit a reaction, may become a permanent memory, may be ignored, etc. [56]. Baddeley 7 8,9 ] developed the theory of working memory that involves the "central executive" - an attention-controlling sub-system, the "visuospatial sketch pad" which manipulates visual images, and the "phonological loop" which stores and rehearses speech-based information.

While very large amount of information comes into sensors, the mind is capable of processing only a limited amount of new information. But, the mind is also very good at on-the-fly abstraction, and mapping of those abstractions onto a huge pool of encoded information in its permanent memory. "Chunks" are associated with the working memory throughput. They were originally proposed by Miller [1011], and there appears to be a limited number (between 3 and 7) that a human can handle/manage at any one time without making an increasing 
number of mistakes. For example, humans may have trouble tracking (without error) more than four to five visual or auditory "targets".

Humans appear be able to enumerate quickly up to about five items, beyond that the process is slower and time taken increases linearly 12. For example, counting 50 stars on a flag may take some time, recognizing a flag takes much less than a second, and retrieving the number of stars that should be on it from permanent memory is also very quick. Humans also appear to handle about four dimensions [13] and up to 5-way interactions reasonably well [14. All this indicates that it is important to manage complexity of content and interrelations presented to humans [13 15]. Beyond a comfortable limit (and that limit may change with age) errors start happening. Most recent examples of sometimes fatal real-time analytics distractions to humans come from the use of cell phones and other in-car devices by drivers (see e.g., [21 22] ).

"Chunks" can contain a very small amount of information - from a single tone, to a very detailed and rich visual, auditory and tactile scene. They can contain raw data, but more often they tend to be abstractions, pattern mappings of complex objects or scenes onto however this impression is stored/encoded in the permanent memory of the subject. Different people very likely extract storable abstractions of the same scene, object, or signal differently. Pattern or "symbol" recognition appears to be the primary way we think about scenes and situations which would otherwise overload our working memory, and the question is how long does it take to map a sensory "chunk" or moment onto a familiar pattern to possibly elicit a conscious reaction.

Chunks also appear to have an expiration time - they are retained in working memory for not more than several minutes and, unless processed through action or permanent memory storage, they are lost. They appear to be constructed from segments that do not exceed about $100 \mathrm{~ms}$ each (but could be shorter). Some classical work in that domain was done by Stroud [23|24] who noted that humans appear to have ability to consciously process from about 5 to 20 of such discrete psychological time slices and effect discriminations based on that. In a situation where a reaction to such a slice is binary (e.g., yes or no, or ok, not ok) conscious absorption and reactions rate appears to be in the 5 to 20 bits per second range. However, in order to match a particular pattern (which could consist of more than one "psychological moment"), mind may need to do a large number of comparisons. This number is sometimes known as the Stroud number, and has been used in attempts to describe software development and software fault generation processes, e.g., [2526]. While 5 or 20 bits may not look like much, to match a 20 bit pattern, for example, may require comparison with as many as 1,000,000 or so internal patterns.

\subsection{Limitations}

Unfortunately, there appear to be at least three bottlenecks on the input path. For example: i) attentional blink (AB), ii) visual short-term memory capacity (VSTM), and iii) psychological refractory period (PRP) phenomena [11. Attention blink relates to the following example, "when subjects attempt to identify 
two targets in a rapid, serial visual presentation of distractors, they are severely impaired at detecting the second of the two targets when it is presented within $500 \mathrm{~ms}$ of the first target [25]. Importantly, the deficit with the second target (T2) is a result of attending to the first target (T1): subjects have no difficulty in reporting T2 when only it is required to be detected" [1115. Part of the problem may lie in the need to move eyes from one spot to another [12 27]. This may also be related to "change blindness" 516]. VSTM (or working memory) capacity of about 4 to 7 items has already been mentioned. PRP is related to taking action as a result of a stimulus. This appears to impact our ability to simultaneously take two actions based on a single stimulus (parallelism).

\section{Systematic Bias}

By nature of their cultural upbringing, educational training, experiences, motivation, trust, religion, politics, perceived and real situational awareness and other mental models, prevailing policies, regulations and laws, etc., humans will often ignore data and information and make conclusions based on other factors, or they might interpret the same information differently. This is often called cognitive bias (see e.g., 2[17]). The list of possible cognitive biases is very long, e.g. [18, and as expected, there are supporters and critics, e.g., [19]. Of course, the issue of systematically biasing information, or its interpretations, to fit a particular purpose has been around since the dawn of time. An iconic phrase in this context is "lies, damned lies, and statistics", e.g. [20], which, as large amounts data become more accessible over internet, is being increasingly used and re-used in many forms. The concept takes an additional dimension when deception is considered in the context of information technology security (see e.g., [28]).

Undeniably, epistemic uncertainty is a very serious issue when doing analytics, and particularly real-time analytics. Ideally, one would like to reduce epistemic uncertainty to aleatory uncertainty.

\section{Summary}

In general, it seems to be well established that humans have a (very) limited ability to pro-actively process new incoming information and act upon it, and a very high propensity to unconscious or conscious misinterpretation and misrepresentation of new information. Implications on analytics are substantial. Careful studies need to be made to develop appropriate methods for reduction of epistemic uncertainty down to aleatoric, and to allow reduction of new incoming information to a level appropriate for human consumption. This includes training of the analysts to work at the appropriate level of abstraction, developing appropriate computer-based tools and approaches, and for both humans and machines to handle unexpected anomalies in (large) input streams. How to do that appears to be an open question, although ideas abound. 
Acknowledgements. This work has been supported in part by the DOE SciDAC grant DE-FC02-07-ER25809, by the U.S. Army Research Office (ARO) grant W911NF-08-1-0105 managed by NC State University Secure Open Systems Initiative, by the IBM Shared University Research Program, and by NSF grants CCF-0939081 and CNS-0910767.

\section{References}

1. Barreto, R., Klasky, S., Mouallem, P., Podhorszki, N., Vouk, M.: Collaboration Portal for Petascale Simulations. In: Proceedings of the International Conference on Collaboration Technologies and Systems, pp. 384-393 (2009)

2. Gilovich, T., Griffin, D.: Heuristics and Biases: Then and Now. In: Gilovich, T., Griffin, D., Kahneman, D. (eds.) Heuristics and Biases: The Psychology of Intuitive Judgment, pp. 1-4. Cambridge University Press (2002)

3. WellPoint and IBM Announce Agreement to Put Watson to Work in Health Care (2011), http://www-03.ibm.com/press/us/en/pressrelease/35402.wss (accessed October 31, 2011)

4. Leherl, S., Fischer, B.: The Basic Parameters of Human Information Processing: Their Role in the Determination of Intelligence. Person. Individ. Diff. 9(5), 883-896 (1988)

5. Thompson, R.F., Madigan, S.A.: Memory. Princeton University Press (2005)

6. Atkinson, R.C., Shiffrin, R.M.: The Control of Short-term Memory. Scientific American 225, 82-90 (1971)

7. Baddeley, A.D., Hitch, G.J.: Working Memory. In: Bower, G.A. (ed.) Recent Advances in Learning and Motivation, pp. 47-89. Academic Press, New York (1974)

8. Baddeley, A.D.: Working Memory. Science 255(5044), 556-559 (1992)

9. Baddeley, A.D.: Working Memory: Looking Back and Looking Forward. Nature Reviews Neuroscience 4, 829-839 (2003)

10. Miller, G.A.: The Magical Number Seven, Plus or Minus Two: Some Limits on our Capacity for Processing Information. Psychological Review 63, 81-97 (1956)

11. Marois, R., Ivanoff, J.: Capacity Limits of Information Processing in the Brain. Trends in Cognitive Sciences 9(6), 296-305 (2005)

12. Piazza, M., Mechelli, A., Butterworth, B., Price, C.J.: Are Subitizing and Counting Implemented as Separate or Functionally Overlapping Processes? NeuroImage 15, 435-446 (2002)

13. Halford, G.S., Wilson, W.H., Phillips, S.: Processing Capacity Defined by Relational Complexity: Implications for Comparative, Developmental, and Cognitive Psychology. Behavioral and Brain Sciences 21, 803-865 (1998)

14. Halford, G.S., Baker, R., McCredden, J.E., Bain, J.D.: How Many Variables Can Humans Process? Psychological Science 16(1), 70-76 (2005)

15. Lavie, N.: Distracted and Confused? Selective Attention Under Load. Trends in Cognitive Sciences 9(2), 75-82 (2005)

16. Simmons, D.J., Levin, D.T.: Change Blindness. Trends in Cognitive Sciences 1(7), 261-267 (1997)

17. Kahneman, D., Tversky, A.: Subjective Probability: A Judgment of Representativeness. Cognitive Psychology 3(3), 430-454 (1972)

18. List of Cognitive Biases (2011), http://en.wikipedia.org/wiki/List_of_cognitive_biases (accessed October $22,2011)$ 
19. Gigerenzer, G.: Bounded and Rational. In: Stainton, R.J. (ed.) Contemporary Debates in Cognitive Science, p. 129. Blackwell (2006)

20. Twain, M.: Chapters from My Autobiography. North American Review DXCVIII. Project Gutenberg (1906), http://www.gutenberg.org/files/19987/19987.txt (retrieved May 23, 2007)

21. Strayer, D.L., Drews, F.A.: Cell-Phone-Induced Driver Distraction. Current Directions in Psychological Science 16(3), 128-131 (2007)

22. Statistics and Facts About Distracted Driving (2011), http://www.distraction.gov/content/get-the-facts/ facts-and-statistics.html (accessed December 05, 2011)

23. Stroud, J.M.: The Fine Structure of Psychological Time. In: Quastler, H. (ed.) Information Theory in Psychology, pp. 174-207. The Free Press, Glencoe (1955)

24. Stroud, J.M.: The Fine Structure of Psychological Time. Annals of the New York Academy of Sciences 138, 623-631 (1967)

25. Halstead, M.H.: Elements of Software Science. Operating and Programming Systems Series. Elsevier Science Inc., New York (1977)

26. Coulter, N.S.: Software Science and Cognitive Psychology. IEEE Transactions on Software Engineering 9(2), 166-171 (1983)

27. Rousselet, G.A., et al.: How Parallel is Visual Processing in the Ventral Pathway? Trends in Cognitive Sciences 8, 363-370 (2004)

28. Yuill, J.: Defensive Computer-Security Deception Operations: Processes, Principles and Techniques. PhD Dissertation, North Carolina State University (2006) 